\title{
Diagnosis of Melanoma Based on the Sparse Auto- Encoder for Feature Extraction
}

\author{
Nadia Smaoui Zghal ${ }^{1^{*}}$, Marwa Zaabi ${ }^{2}$ and Houda Derbel ${ }^{3}$ \\ ${ }^{1}$ CEM Laboratory, ENIS, Sfax University, Tunisia. \\ ${ }^{2}$ CEM Laboratory, ENIG, Gabes University, Tunisia. \\ ${ }^{3}$ CEM Laboratory, FSS, Sfax University, Tunisia.
}

\begin{abstract}
Authors' contributions
This work was carried out in collaboration among all authors. Author NSZ designed the study, performed the statistical analysis, wrote the protocol, and wrote the first draft of the manuscript. Authors $M Z$ and $H D$ managed the analyses of the study. Author $M Z$ managed the literature searches. All authors read and approved the final manuscript.

Article Information

DOI: $10.9734 / A R R B / 2020 / v 35 i 1230327$ Editor(s):

(1) Dr. David E. Martin, DFH Pharma Inc., Gaithersburg, Reviewers:

(1) Pradeoth Mukundan Korambayil, Kerala University of Health Science, India. (2) Jufriadif NA`am, Universitas Putra, Indonesia. Complete Peer review History: http://www.sdiarticle4.com/review-history/64708
\end{abstract}

Original Research Article

Received 25 October 2020

Accepted 30 December 2020

Published 31 December 2020

\section{ABSTRACT}

Aims: Skin cancer is a fairly critical disease all over the world and especially in Western countries and America. However, if it is perceived and treated early, it is quite often curable. The main risk factors for melanoma are exposure to UV rays, the presence of many moles, and heredity. For this reason, this work focuses on the issue of automatic diagnosis of melanoma. The aim is to extract significant features from pixels of the images based on an unsupervised deep learning technique which is the sparse autoencoder method.

Methodology: A preprocessing phase is required to remove the artifacts and enhance the contrast of the images before proceeding with the feature extraction. Once the characteristics are extracted automatically, the support vector machine classifier and the k-nearest neighbors are applied for the classification phase. The objective is to differentiate between 3 categories: melanoma, suspected case, and non-melanoma. Finally, the $\mathrm{PH} 2$ database is used to test the proposed approaches (200 images are presented in this dataset: 80 atypical nevi, 80 common nevi, and 40 melanoma).

Results: The obtained results in terms of specificity, accuracy, and sensitivity present noticeable 
performances with the support vector machine classifier (achieved $94 \%$ overall accuracy) and the k-nearest neighbors (92 \%).

Conclusion: This study's experimental findings showed that the best performance was obtained by the approach based on a deep sparse autoencoder combined with support vector machine.

Keywords: Melanoma; sparse autoencoder; support vector machine; k-nearest neighbors.

\section{INTRODUCTION}

Melanoma is considered the most insecure form of skin cancer. It starts in the melanocytes found in the outer layer of the skin, which are pigmentproducing cells. Melanoma is caused by sunlight or tanning ultraviolet radiation that produces genetic deficiency, which the skin cells are rapidly expanded and developing malignant tumors [1]. Different experts have concluded that approximately $90 \%$ of melanomas are caused by extreme UV exhibition and sunburn [2]. Malignant melanoma, with a ten-fold higher occurrence rate, is expected to become one of the most common malignant tumors in the future. Indeed, the incidence of malignant melanoma has raised dramatically since 1970; for example, in the USA, this occurrence increases annually by around $4 \%$ [2]. The main reason for the rapid increase in melanoma cases is prolonged exposure to ultraviolet (UV) radiation from the sun (for example the sunbeds).Dermatological oncology is beginning to be a very significant field of medicine due to the high incidence of skin cancer.

Today, progress is evident both in primary research on tumor pathogenesis (the role of viruses or genes in tumor growth) also in the improvement of effective computer-aided diagnostic algorithms to assist dermatologists and in particular, family doctors because they have difficulties proper and visual diagnosis of skin lesions.

In lasted studies, multiple methods for the detection of melanoma have been suggested. For example, in [3], the proposed method describes the identification and segmentation of Melanoma utilizing hill climbing and Fuzzy C Means (FCM). The procedure started with image conversion from RGB space to CIELab space. CIELab image's 3D histogram furnish seeds for FCM. For the segmentation task, they function as initial clusters. Also, in [4], with Gaussian Filter and FCM, the automatic detection and segmentation approach is suggested. The authors in [5] proposed a method contains different steps: (1) preprocessing, (2) threshold segmentation, (3) extraction of features (utilizing Gray Level Co-occurrence Matrix GLCM), (4) selection of features (with Principal Component Analysis PCA), (5) classification with SVM. Besides, the method in [6] includes an automatic ABCD technique of dermoscopy lesions images (asymmetry, border, color, and diameter). Preliminary, in a preprocessing phase, they used hair removal that is based on morphological filtering and thresholding. The lesions are eventually classified (melanoma or benign). Also, the authors in [7] used the technique ABCD and they applied the neural networks for classification. In addition, two methodologies for detecting melanoma are outlined in [8]. The initial technique describes the detection of skin lesion melanoma based on Total Dermoscopic Score (TDS) at different levels utilizing ABCD characteristics. The second technique describes multiple types of skin cancers. For the early detection of melanoma, the authors in [9] suggested a non-invasive automated skin lesion analysis method. For successful classification and extraction of skin wound characteristics, hair identification and removal are carried out. A rapid marching algorithm in painting is applied for hair removal. In [10], for the early detection of melanoma, an automated diagnostic algorithm has been implemented. To evaluate a full study of concave borders, a novel boundary descriptor is suggested. To form a comprehensive feature vector, the Fourier descriptors are connected with a set of shape and textural characteristics. In this work, as a classifier, they used the SVM. The authors in [11] proposed a method that contains many stages. Initially, the preprocessing step is done. Next, an image segmentation technique is used with the method of Otsu thresholding, followed by extraction of the feature. Then, in order to identify the skin image, classification using the SVM is performed. Also, in [12], the authors established an automated detection method for melanoma. In order to automatically segment and extract the borders of the affected area, a mix of Otsu and kmeans clustering segmentation methods are applied. Moreover, along with color and texture, they extracted various non-linear features. With a machine learning model compound of 5 different 
classifiers, the efficacy of these characteristics was predicted. In [13], the authors proposed an approach based on a combination of texture and color feature extraction. Firstly, by translating the images into Grayscale by the principal component analysis (PCA) technique and the multilevel Otsu thresholding, normal and diseased images are collected and preprocessed. Then, shape, color, and texture characteristics are extracted and the SVM classifier is used for classification. Moreover, in [14], the suggested approach involves (1) preprocessing, (2) GrabCut technique, derived from image segmentation graph cuts, (3) image smoothing, (4) sequential backward selection (SBS) technique, (5) k-nearest neighbors (KNN) algorithm for classification. The authors in [15] proposed a system for the detection of melanoma. Initially, the preprocessing is introduced. The Active Contour segmentation method is then used to extract the region of interest. From the segmented portion, various color characteristics have been extracted and the output of the system is tested using 3 classifiers (KNN, decision tree, and naive bayes). In [16], for skin lesion segmentation, they used a regionbased, fully-automatic algorithm. Two parallel skin detection processes (using thresholding) and lesion segmentation (it is organized in 2 stages: Edge Detection and Delaunay Triangulation) are applied. The first is utilized to divide the image into local coherent regions. The second is used to incorporate homogeneous regions in a global fashion. After that, merge the results to obtain an appropriate binary mask contain the area of the lesion. Besides, a novel approach is proposed in [17]: the value of depth is illustrated in melanoma skin lesions. A novel $3 \mathrm{D}$ reconstruction algorithm has been suggested to extract the depth. Using the adaptive snake algorithm, segmentation is accomplished. Therefore, the inclusion of the depth feature has allowed the classification rate to increase markedly. The authors in [18] suggested a method based initially on the reduction of the noise utilizing the Dull-Razor software, then the segmentation utilizing an automated segmentation process. Using the Grey Level CoOccurrence Matrix (GLCM) and simple statistical methods, the features are then extracted. Then, these features are introduced into the support vector machine (SVM). In [19], Image acquisition, hair detection and removal, active contour-based segmentation, feature extraction using color correlogram and texture, and Bayesian classifier for classification are the key components of the proposed method. In addition, for melanoma image classification, the authors in [20] proposed a deep convolutional neural network (DCNN). It allows to automatically classify the melanoma images. There are several sets of convolutional layers and max-pooling layers in the proposed network architecture, followed by a drop out layer and a fully-connected layer. In [21], the authors presented the algorithm You Just Look Once (Yolo), which is based on DCNN. The Yolo algorithms consist of YoloV1, YoloV2, and YoloV3, the methodology first resets the size of the input image, and second splits the image into multiple cells. The network will try to predict the bounding box of the object and the class value, depending on the location of the detected object in the cell. Also, in [22], to automatically detect melanoma, they used a real-time object detection process. A state-of-the-art YoloV2 detection model is used, to detect melanoma in real-time (the YoloV2 utilizes a single full-image neural network, allowing for real-time performance). The authors in [23] implemented a CNN using Aggregated Transformation and Squeeze-andExcite mechanisms combined into a residual processing block (the architecture is built and trained from scratch). Also, to detect melanoma, the authors in [24] suggested an approach based on residual-squeeze-and-excitation (RSE). Precisely, Inception-v4 architecture is used to obtain the spatial characteristics and increase the variety of functions. Then, for the skin lesion classification, they used the SVM. Besides, in [25], the approach consists of 3 steps: preprocessing to remove hair utilizing morphological operations, melanoma lesion localization using a DCNN called SingleShot Detection (SSD) network, and segmentation using the algorithm level set. Moreover, the authors in [26] indicated that high-quality diagnosis for skin lesion diseases can be predicted with the deep learning method. By adding the adaptive piecewise linear (APL) function, they built a DCNN for classification. In [27], the authors suggested a Deep Attention Network (DAN). To learn image characteristics, it utilizes the focus module. A bottom-up and topdown structure and a convolutional feed-forward architecture compose the attention module. to learn specialized characteristics. They restricted DAN's loss function on the basis of the Fisher criterion, in this way, they obtained the optimal classification value. Furthermore, to detect lesion attributes of melanoma automatically, the authors in [28] proposed a multi-task U-Net model. The network has 2 stages, the first is the classification and the second is the segmentation to segment the attributes in the images. In [29], 
the authors also proposed deep learning. Using the same dataset with data augmentation, they constructed numerous deep learning models. DCNN including ResNet-152, DenseNet161, and Inception-v4 is trained for the classification of melanoma. U-Net and UNet with VGG-16 are also trained for lesion segmentation.

In this paper, we suggest a novel method for diagnostic of melanoma. More precisely, the strategy is based initial on the extraction of a feature vector from dermoscopic images and then the classification with the SVM. to classifying them. To remove unnecessary objects and enhance the contrast of the images, these stages are preceded by pretreatment. The extraction of features from preprocessed images is carried out with a Sparse Autoencoder that forms a vector called image signature. The signatures are utilized to identify 3 populations of melanoma, suspicious and non-melanoma which are introduced as entry in the SVM classifier and the KNN.

The remainder of the paper is organized as follows: An outline of the Sparse Autoencoder strategy is given in Section 2. The proposed method is defined in section 3 . Section 4 discusses the experimental findings and section 5 presents a comparative analysis. The conclusion ends the paper.

\section{MATERIALS AND METHODS}

Centered on deep learning, our method of classifying skin lesions in dermoscopy images is discussed in this section. The initial phase is to preprocess the images and then extract features founded by the Sparse Autoencoders and eventually classify the images utilizing the SVM.

In this section, the steps involved in applying our method to the $\mathrm{PH} 2$ dataset developed by the ADDI (Automatic computer-based Diagnostic system for Dermoscopy Images) project are presented [30]. The dermoscopic images were collected at the Dermatology Department of the Pedro Hispano Hospital (Matosinhos, Portugal) using the Tuebinger Mole Analyzer system with $20 x$ magnification. All the images, which are in 8bit RGB color with a resolution of $768 \times 560$ pixels, were taken under the same conditions. 200 images are presented in this dataset (40 melanoma, 80 common nevi, and 80 atypical nevi). The $\mathrm{PH} 2$ database is divided into two sets : training and testing $(75 \%$ used for the training and $25 \%$ used for testing).
Fig. 1 presents several images of dermatoscopic of the three groups, and Fig. 2 displays a flowchart of the proposed method.

\subsection{Preprocessing Phase}

Because of numerous interference sources and many phenomena that influence the process of image acquisition, dermoscopic images are often degraded by noise. They also contain foreign artifacts (skin lines, hairs, air bubbles). In order to enhance the quality and to reduce the noise and the number of artifacts, image enhancement techniques are therefore necessary for dermoscopic images. This makes it easier to detect the image feature and also extract it with an automated image processing model [31]. The phase of preprocessing is split into 3 steps. Initially, the median filter, which is an important noise reduction tool, is applied. A morphological closure is then used to remove all remaining artifacts after filtering. (such as hairs). Finally, a contrast improvement is applied, providing an understanding of the lesion and subsequently a better interpretation. Fig. 3 present some examples of preprocessed images.

\subsection{Feature Extraction}

The techniques of deep learning allow machines to learn from massive datasets. Input datasets (medical images), are explicitly categorized by these models automatically. They allow to give fine and up-to-date classifications that, in certain cases, can overcome human assessment. In several domains, deep learning models produce unprecedented outcomes, for this reason, they considered powerful models in order to discover complex data relationships and to detect highlevel abstractions. The network depth allows to extract pertinent characteristics holding some intrinsic value for the classification task.

\subsubsection{Autoencoder structure}

Autoencoder is a type of unsupervised learning structure owning three layers which are the input layer, the hidden layer, and the output layer as presented in Fig. 4. The training process of the autoencoder is based on two parts which are the encoder and the decoder. The former is used for the map of the input data into the hidden representation, and the second is regarded as the reconstruction of the input data from the hidden representation [32]. 

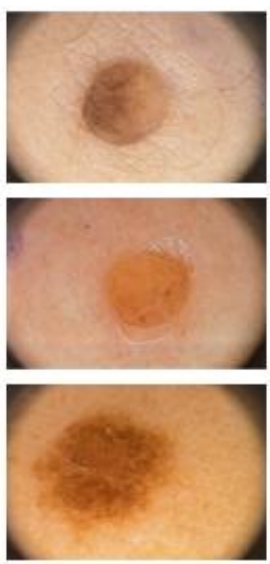

a)
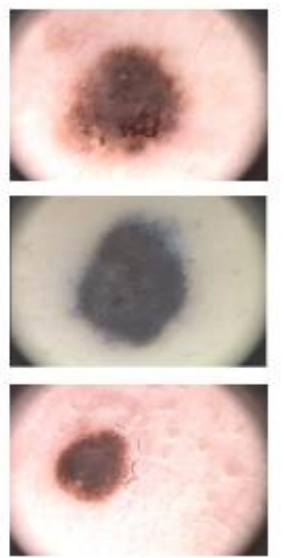

b)
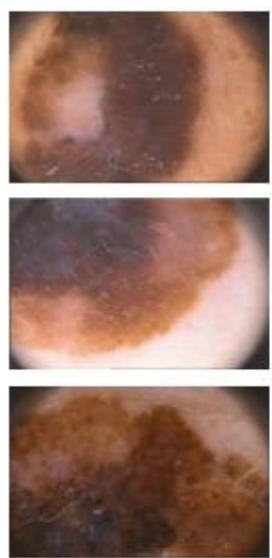

c)

Fig. 1. PH2 database sample lesions presentation: a) Atypical Nevus, b) Commun Nevus, c) Melanoma

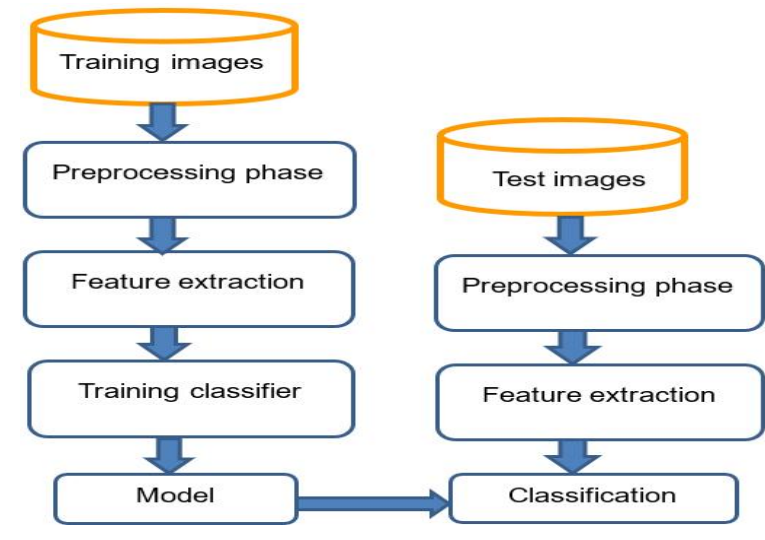

Fig. 2. Flowchart of the proposed system

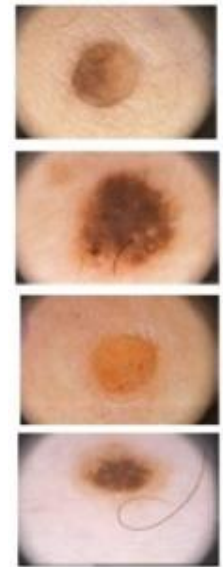

1)
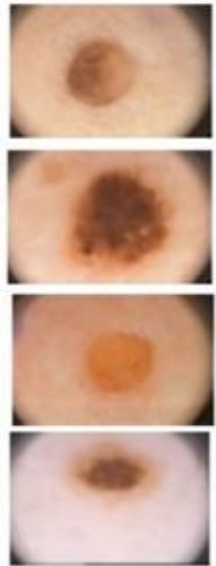

2)

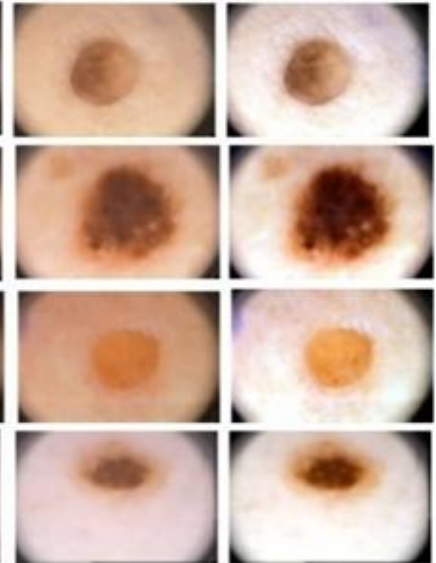

3)

4)

Fig. 3. Sample dermoscopy images: 1) Original images, 2) Median filter, 3) Morphological closing, 4) Image enhancement 


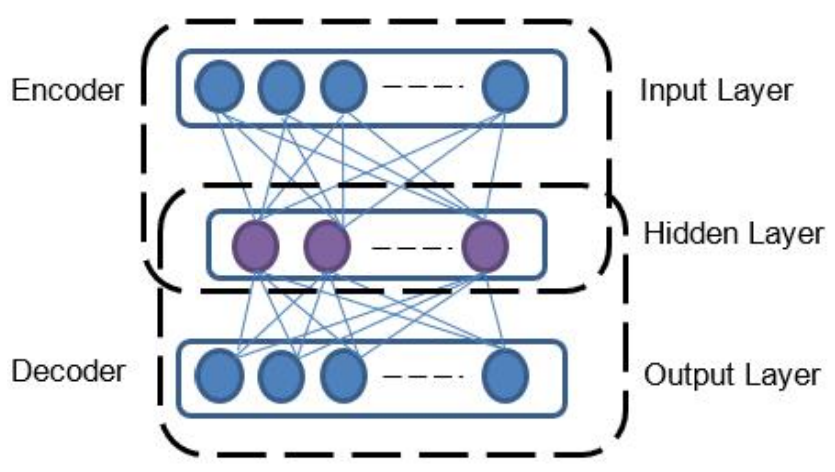

Fig. 4. Autoencoder structure

We consider the unlabeled input dataset $x_{n} \in$ $R^{m+1}, h_{n}$ is the hidden encoder vector which is calculated from $x_{n}$ and $y_{n}$ represents the output layer's decoder. The following equations represent respectively the encoding process and the decoding process:

$$
\begin{aligned}
& h_{n}=f e\left(\omega_{1} x_{n}+b 1\right) \\
& y_{n}=f d\left(\omega_{2} h_{n}+b 2\right)
\end{aligned}
$$

Where $f e$ and $f d$ are respectively the encoding and the decoding function. $\omega_{1}$ and $\omega_{2}$ are respectively the weight matrix of the encoder and the decoder. $b_{1}$ and $b_{2}$ are the bias vectors.

The parameters of the autoencoder are optimized in order to minimize the reconstruction error as presented by the following equation.

$$
\emptyset(\theta)=\underset{\theta, \theta^{n}}{\arg \min _{1}} \sum_{i=1}^{n} L\left(x^{i}, y^{i}\right)
$$

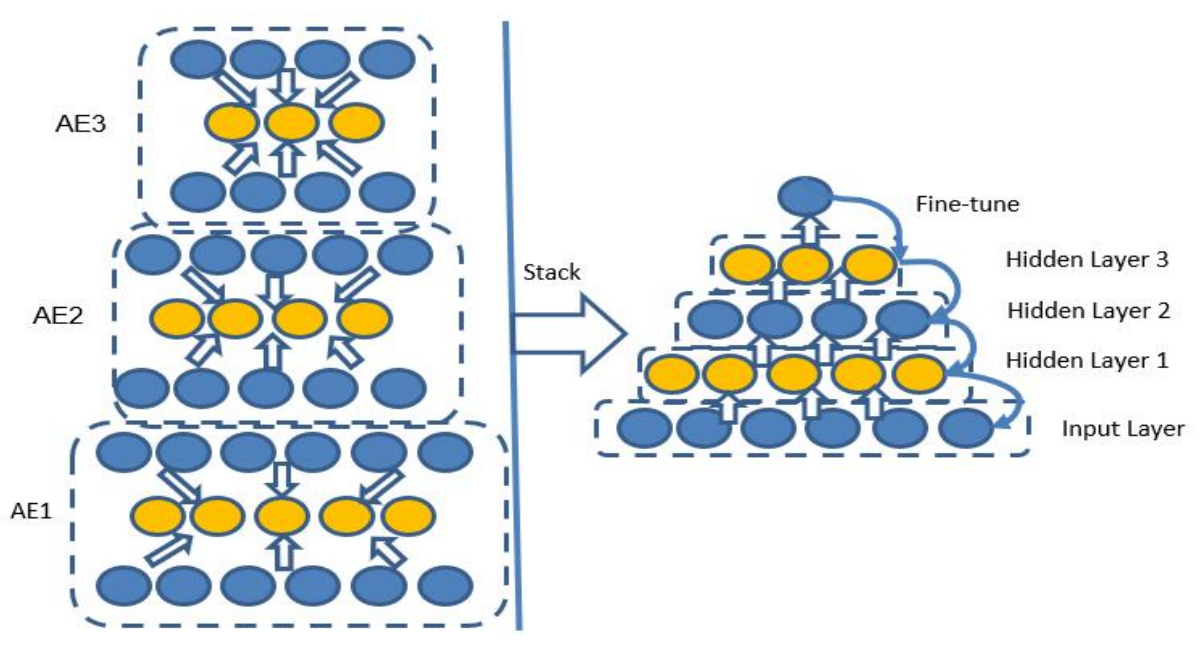

Fig. 5. Stacked autoencoders
Where $L$ is the loss function presented by $L(x, y)=\|x-y\|^{2}$

\subsubsection{Stacked autoencoders}

Fig. 5 presents the stacked autoencoders (SAEs) structure that It consists of various layers that the output of each layer is related to the inputs of the follow layer, so that each layer try to reconstruct the output of the precedent layer. The technique of SAEs can be presented in three steps; (i) The first autoencoder is trained by the input data to obtain the first learned feature vector. (ii) The obtained feature vector is then used as the input for the second layer to get the second learned feature vector. This process is repeated until the training ends. (iii) After finishing the training of all the hidden layers, a backpropagation algorithm is used for minimizing the cost function and for updating the weights to achieve finetuning [32]. 
In our research, the objective is to use the training images from the $\mathrm{PH} 2$ database (150 images) to train the Autoencoder and classify then the test set (50 images) utilizing the SVM. For that, the $\mathrm{PH} 2$ dataset images are resized to $128 * 128$ in order to promote the learning process. Second, we begin by training the initial layer, on the training data, without using labels. The number of neurons is made smaller than the input size in the hidden layer in order that the first Autoencoder learns a compact representation of the input. In fact, to learn a sparse representation, the Autoencoder uses regularizes. Therefore, an adjustment of the Autoencoder parameters is necessary to be able to track the impact of the regularizes on the learning phase. The second auto-encoder is similarly trained. The primary difference is that the characteristics which were extracted from the first Autoencoder are the training data in the second Autoencoder.Also, the size of the hidden representation is diminished. Thus, in the second Autoencoder, the encoder can learn a smaller representation of the input data. We can extract a second set of characteristics in this way. In this paper, the work discussed is based on the fundamental model implementing multiple layers. A powerful model is described in the experimental section.

\subsection{Classification}

The Autoencoder enables each image to generate a unique vector representation $y$ in this section. This $y$ signature has the same dimension for all images and serves as a global and coherent representation. To assign a label to the signature $y$, a good classifier is also required.

In this article, the first objective is to classify the images based on SVM classifier(a supervisedlearning model). The SVM algorithm is founded on creating a system that assignsa novel sample the corresponding class. The SVM model is a configuration of samples in space as points; a linear function is applied in such a way that a clear gap segregated all the samples of the various groups [33]. The SVM tries to get the best hyperplane for classification into distinct classes. Thus, the SVM classifier have for objective to split the learning examples into 3 classes (suspicious, non-melanoma, and melanoma). Second, another supervised method is applied to distinguish these three classes, it is the KNN. Their theory implies that an object (of undefined class) is compared all the others objects in data. Indeed, the algorithm seek the $\mathrm{k}$ nearest neighbors of the novel object to classify in term of the distance, as the Euclidean distance and attribute the class with the smallest distance to this object [34].

\section{RESULTS AND DISCUSSION}

\subsection{Classification Assessment}

Classification performance was measured in terms of precision, sensitivity and specificity [35]. The specificity indicates how effective the test for the detection of normal patients. The sensitivity indicates how effective the test is for disease detection, and precision is presented as the proportion of correct test results. These parameters are presented in terms of true positives (TPs), true negatives (TNs), false positives (FPs), and false negatives (FNs) in the following equations.

$$
\begin{aligned}
& \text { Specificity }=\frac{T N}{T N+F P} \\
& \text { Sensitivity }=\frac{T P}{T P+F N} \\
& \text { Precision }=\frac{T P}{T P+F P}
\end{aligned}
$$

\subsection{Simulation Results}

In MATLAB 2017b, simulations are conducted on the Microsoft Windows 10 operating system, with Intel (R) Core (TM) i3 4 GB RAM and $2.5 \mathrm{GHz}$ $\mathrm{CPU}$ processors. The autoencoders is trained, layer by layer, in an unsupervised manner, as presented in section 2.This training is repeated various times by changing its parameters in order to define the number of neurons in a hidden layer and the parameters of regulation until those that give a minimal reconstruction error. Table 1 presents the parameters utilized for the simulation of our model.

Table 1. Simulation parameters

\begin{tabular}{ll}
\hline Parameters & Values \\
\hline MaxEpochs & 1000 \\
L2Weight Regularization & 0.004 \\
Sparsity Regularization & 4 \\
Sparsity Proportion & 0.15 \\
Learning Rate & 0.1 \\
\hline
\end{tabular}

As main factors influencing the efficiency of our model, the number of hidden layers should be taken into account. With various numbers of hidden layers, we conducted the experiment as 
presented in Fig. 6. A sparse Autoencoder with three hidden layers including 100,50 , and 20 neurons respectively combined with a linear kernel SVM gives the best result. Indeed, with the number of added layers, the classification rate increases gradually to achieve a maximum level of three layers.

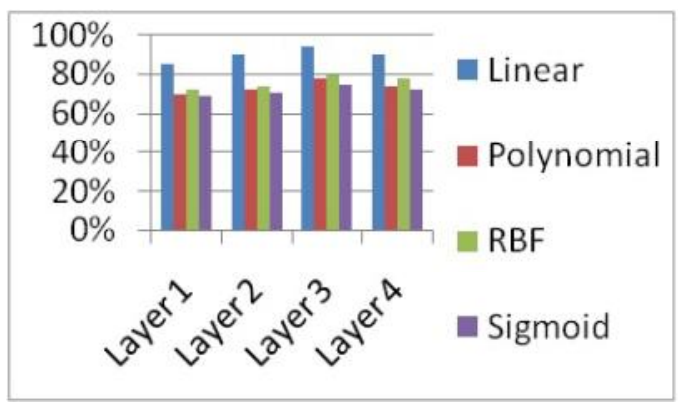

Fig. 6. Classification rates obtained for different types of SVM kernels

\subsection{Evaluation}

The proposed approach in this study point to classify dermatoscopic images into 3 classes: suspicious lesion, non-melanoma, and melanoma. This method is based on an Autoencoder's extraction of features. Also, the classification is carried out by a linear kernel SVM.
With test images from the $\mathrm{PH} 2$ database, the simulations were realized. The classification performance results are presented in the Table 2.

The values presented in the Table 2 show the efficiency of the proposed system.

The second idea of evaluating the proposed system was presented by just changing the classification technique using the KNN. For that, the extracted features from the considered model (Autoencoder) are fed into the KNN to make a comparison with the SVM model.

Table 3 presents the classification rate for different $\mathrm{k}$ values.

From this table which presents the performance rates for different $k$ values, the best result is found for $k=5$. Although this value shows that the technique used (Autoencoder $+\mathrm{KNN}$ ) is a good technique to classify the 3 classes (suspicious, non-melanoma, and melanoma), it still remains inferior to the proposed technique (Autoencoder + SVM) (see Fig. 7).

Finally, A comparative analysis is conducted based on performance rates (as illustrate by Fig. 7) so that evaluate our approach relative to other recent methods. An automated melanoma detection system for dermoscopy images founded on multi-scale lesion bias representation

Table 2. Classification Rates for different classes

\begin{tabular}{lllll}
\hline Classes & Sensitivity & Specificity & Precision & Overall accuracy \\
\hline Non-Melanoma & $95 \%$ & $52.5 \%$ & $95 \%$ & \\
Suspicious & $95 \%$ & $96 \%$ & $95 \%$ & $94 \%$ \\
Melanoma & $90 \%$ & $66.66 \%$ & $90 \%$ & \\
\hline
\end{tabular}

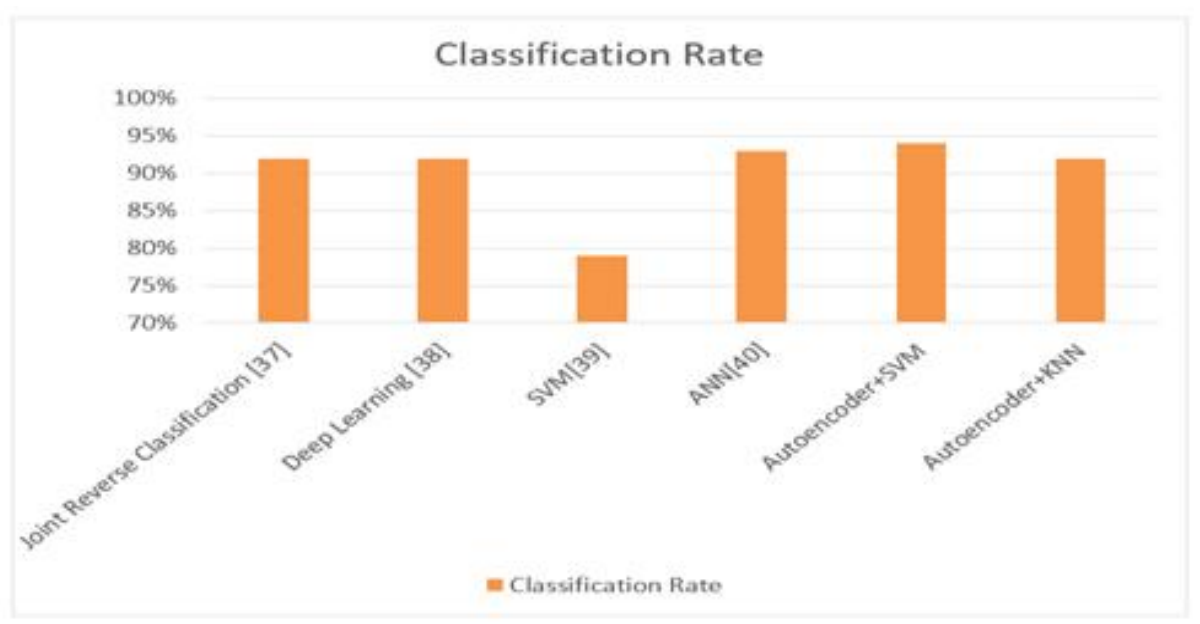

Fig. 7. Comparative study 
(MLR) and joint reverse classification (JRC) is described in the study proposed in [36]. Combined with JRC, the MLR representation enables a collection of closely related histograms to be used to extract additional details for melanoma detection. In [37], for the diagnosis of Melanoma, the authors suggested using the Convolutional Neural Network. In [38], the authors used a technique focused on texture and color characteristics to detect melanoma in dermoscopy images. Moreover, four machine learning techniques: SVM, Decision Tree, KNN, and ANN were used in the work presented in [39]. The classification rates are $89.5 \%, 90 \%$, $82 \%$, and $92.5 \%$ respectively. For our analysis, a comparison is made using only ANN that has the best accuracy. The $\mathrm{PH} 2$ database is the foundation of all these studies.

The proposed approach with the SVM has a higher classification rate value compared to the literature studies presented in Fig. 7. Using a larger number of images, this result can be further ameliorated. Furthermore, this research can be used in many medical problems to make autonomous decisions.

Table. 3 Classification rates for different $\mathbf{k}$ values

\begin{tabular}{ll}
\hline K value & Classification rate \\
\hline $\mathrm{K}=1$ & $86 \%$ \\
$\mathrm{~K}=3$ & $88 \%$ \\
$\mathrm{~K}=5$ & $92 \%$ \\
\hline
\end{tabular}

\section{CONCLUSION}

An efficient approach to the automatic diagnosis of melanoma based on a sparse autoencoder combined with a classifier is proposed in the presented work. The developed approach is based first on pretreating the dermatoscopic images in order to remove noise and enhance their contrast. After that, features are extracted based on asparse autoencoder algorithm. Those features form a signature utilized for classification for each image. Finally, for classification, the extracted features are fed into the SVM algorithm, and the KNN algorithm. The experimental results of this study demonstrated that with a $94 \%$ classification rate, the method based on a sparse autoencoder combined with SVM achieves better efficiency.

\section{COMPETING INTERESTS}

Authors have declared that no competing interests exist.

\section{REFERENCES}

1. Argenziano $\mathrm{G}$, Soyer HP, De Giorgio V, Piccolo D, Carli $P$, Delfino $M$, et al. Interactive atlas of dermoscopy. Milano, Italy. Edra Medical Publishing and New Media. 2000;50(5):807-808.

2. Tadeusiewicz R. Place and role of intelligent systems in computer science. 2010:193-206.

3. Ganesan P, Vadivel M, Sivakumar VG, Vasanth K. Hill climbing optimization and fuzzy C-means clustering for melanoma skin cancer identification and segmentation. ICACCS. 2020;357-36.

4. Chakkaravarthy AP, Chandrasekar A. Automatic detection and segmentation of melanoma using fuzzy C-means. ICONSTEM. 2019;132-136.

5. Alquran $\mathrm{H}$, Qasmieh IA. The melanoma skin cancer detection and classification using support vector machine. AEECT; 2017.

6. Lattoofi NF, Al-sharuee IF, Kamel MY, Obaid $\mathrm{AH}$. Melanoma skin cancer detection based on $A B C D$ rule. CAS. 2019;134-157.

7. Cueva WF, Muñoz F, Vásquez G, Delgado G. Detection of skin cancer "Melanoma" through Computer Vision; 2017.

8. Reshma M, Shan BP. Two methodologies for identification of stages and different types of melanoma detection. ICEDSS. 2017;257-259.

9. Joseph S, Panicker JR. Skin lesion analysis system for melanoma detection with an Effective Hair Segmentation Method. 2016:91-96.

10. Mahdiraji SA, Baleghi Y, Sakhaei SM. BBS, a new descriptor for melanoma/ nonmelanoma discrimination. ICEE. 2018:1397-1402.

11. Mane S, Shinde S. A Method for Melanoma Skin Cancer Detection Using Dermoscopy Images;2018.

12. Munia TK, Alam N, Neubert J, Fazel-Rezai R. Automatic Diagnosis of Melanoma Using Linear and Nonlinear Features from Digital Image. 2017;4281-4284.

13. Pallavi B, Keshvamurthy. A hybrid diagnosis system for malignant melanoma detection in dermoscopic images. RTEICT. 2019;1471-1776. 
14. Mustafa S. Feature Selection Using Sequential Backward Method in Melanoma Recognition; 2017.

15. Shalu, Kamboj A. A color-based approach for melanoma skin cancer detection. ICSCCC. 2018;508-513.

16. Pennisi A, Bloisi D, Nardi D, Giampetruzzi AR, Mondino C, Facchiano A. Melanoma Detection Using Delaunay Triangulation. 27th International Conference on Tools with Artificial Intelligence. 2015;791-798.

17. Satheesha TY, Satyanarayana D, Giriprasad MN, Nagesh KN. Detection of melanoma using distinct features. 3rd MEC International Conference on Big Data and Smart City.2016.

18. Shafiq S, Prasad PWC, Alsadoon A, Ali S, Elchouemi A. Computer aided early detection and classification of malignant melanoma. 10th International Conference on Computational Intelligence and Communication Networks. 2018:92-98.

19. Soumya RS, Neethu S, Niju TS, Renjini A, Aneesh RP. Advanced earlier melanoma detection algorithm using colour correlogram. 2016;190-194.

20. Rokhana R, Herulambang W, Indraswari R. IES. Deep convolutional neural network for melanoma Image Classification. 2020;481-486.

21. Nie Y, Sommella P, O’Nils M, Liguori C, Lundgren $\mathrm{J}$ Automatic Detection of Melanoma with Yolo Deep Convolutional Neural Networks. EHB. 2019.

22. Roy SS, Haque AU, Neubert J. Automatic diagnosis of melanoma from dermoscopic image using real-time object detection. CISS. 2018.

23. Rodrigues JF, Brandoli B, Yahia SA. DermaDL: advanced Convolutional Neural Networks for automated melanoma detection. CBMS. 2020:504-509.

24. Huang R, Liang J, Jiang F, Zhou F, Cheng $\mathrm{N}$, Wang $\mathrm{T}$, Lei B. MelanomaNet: An effective network for melanoma detection. 2019:1613-1616.

25. Rashid F, Irtaza A, Nida N, Javed A, Malik $\mathrm{H}$, Malik $\mathrm{KH}$. Segmenting melanoma Lesion using Single Shot Detector (SSD) and Level Set Segmentation Technique. MACS.2019.

26. Namovoz A, Cho YI. Convolutional neural network algorithm with parameterized activation function for melanoma classification. 2018:417-419.

27. Ma Z, Yin S. Deep attention network for melanoma detection improved by color constancy. 9th International Conference on Information Technology in Medicine and Education. 2018;123-127.

28. Chen EZ, Dong X, Li X, Jiang H, Rong R, Wu J. Lesion attributes segmentation for melanoma detection with multi-task u-net. ISBI. 2019;485-488.

29. Phan HN, Koay CY, Chakraborty T, Gupta $\mathrm{S}$, Tan $\mathrm{BL}, \mathrm{Wu} \mathrm{H}$. Lesion segmentation and automated melanoma detection using deep convolutional neural networks and XGBoost. ICSSE. 2019;142-147.

30. Mendona T, Ferreira PM, Marques JS, Marcal AR,Rozeira J. PH 2-A dermoscopic image database for research and benchmarking, in Proc. 35th Annual International Conference of the IEEE EMBS. 2013;5437-5440.

31. Penissi A, D. D. Bloisi, Nardi D, Giampetruzzi AR, Mondino C, Facchiano A. Skin lesion image segmentation using Delaunay Triangulation for melanoma detection, Comput. Med. Imaging Graph. 2016;52:89-103.

32. Shen D, Wu G, Suk H. Deep learning in medical image analysis. Review in Advance first posted online. 2017;221-247.

33. Cristianini N, Shawe-Taylor J. An Introduction to Support Vector Machines and Other Kernel-based Learning Methods. First Edition. Cambridge University Press; 2000.

34. Zaabi M, Smaoui N. Comparative study of two classification methods for the detection of alzheimer's disease. Current Medical Imaging Reviews. 2017;88-94.

35. Ann J, A, Ruiz C. Using Deep Learning to Detect Melanoma in Dermoscopy Images, International Journal of Machine Learning and Computing. 2018;8:61-68.

36. Bi L, Kim J, Ahn E, Feng D, Fulham M. Automatic melanoma detection via multiscale lesion-biased representation and joint reverse classification. ISBI. 2016; 1055-1058.

37. Salido JA, Ruiz C. Using deep learning to detect melanoma in dermoscopy images. International Journal of Machine Learning and Computing. 2018;8:61-68. 
38. Marques JS, Barata C, Mendonça T. On the role of texture and color in the classification of dermoscopy images.34th Annual International Conference of the IEEE EMBS. 2012;4402-4405.
39. Ozkan IA, Koklu M. Skin lesion classification using machine learning algorithms. International Journal of Intelligent Systems and Applications in Engineering. 2017;5(4):285-289.

(c) 2020 Zghal et al.; This is an Open Access article distributed under the terms of the Creative Commons Attribution License (http://creativecommons.org/licenses/by/4.0), which permits unrestricted use, distribution, and reproduction in any medium, provided the original work is properly cited.

Peer-review history:

The peer review history for this paper can be accessed here: http://www.sdiarticle4.com/review-history/64708 